\title{
AN EXTENSION OF LAGRANGE'S EQUATIONS
}

\author{
BY C. A. SHOOK
}

1. Introduction. In studying the motion of a planet about the sun under the action of a disturbing planet the following coordinates are convenient. Let the plane determined by the origin and the tangent to the planet's path at any instant be $\pi$. Let $x, P, N$ denote respectively the points where a unit sphere about the origin is cut by the $x$ axis, the radius vector to the planet, and the line of intersection of $\pi$ and the $x y$ plane. Then let $\theta=x N, w=N P, \phi=$ the dihedral angle between $\pi$ and the $x y$ plane, and $r=$ radius vector, it being understood that $\phi$ is in the first quadrant and $\theta$ is between 0 and $360^{\circ}$.

If $\beta$ and $\lambda$ denote latitude and longitude respectively, we have for the kinetic energy of a unit particle

$$
2 T=\dot{r}^{2}+r^{2}\left(\dot{\beta}^{2}+\cos ^{2} \beta \dot{\lambda}^{2}\right) .
$$

But we have the relations

$$
\left.\begin{array}{rl}
\sin \beta & =\sin \phi \sin w \\
\tan (\lambda-\theta) & =\cos \phi \tan w
\end{array}\right\},
$$

whence

(3) $2 T=\dot{r}^{2}+r^{2}\left[\left(1-\sin ^{2} w \sin ^{2} \phi\right) \dot{\theta}^{2}+\dot{w}^{2}+\sin ^{2} w \dot{\phi}^{2}\right.$

$$
+2 \cos \phi \dot{\theta} \dot{w}-2 \sin w \cos w \sin \phi \dot{\theta} \dot{\phi}] \text {. }
$$

It will be shown that the condition that the velocity be in the plane $\pi$ is

$$
\frac{\partial T}{\partial \dot{\phi}}=0,
$$

which in the present case reduces to

$$
\sin w \dot{\phi}-\cos w \sin \phi \dot{\theta}=0 .
$$

If by means of this relation we eliminate $\dot{\phi}$ from $T$ we get

$$
2 T=\dot{r}^{2}+r^{2}(\dot{w}+\cos \phi \dot{\theta})^{2} .
$$

Now put $v=w+\theta$ and $\Gamma=1-\cos \phi$, so that 


$$
2 T=\dot{r}^{2}+r^{2}(\dot{v}-\Gamma \dot{\theta})^{2} .
$$

The four coordinates used are $r, \theta, v, \Gamma$.

The question now is whether or not we may use Lagrange's equations with this value of $T$. There are two reasons why it is by no means obvious that we may. In the first place we are using four coordinates and the dynamical system has but three degrees of freedom. In the second place, partial derivatives are involved in Lagrange's equations. Now the partial derivatives of $T$ depend not only on its value but on the way it is expressed. We might, for example, have eliminated $\dot{\theta}$ by means of (4); or we might have assumed a relation altogether different from (4). We shall prove a theorem which shows that, in spite of all this, Lagrange's equations do apply in the present case.

2. Conservative Holonomic Dynamical System. In a conservative holonomic dynamical system with $n$ degrees of freedom let the kinetic energy, $T$, be given by

$$
2 T=a_{i j} \dot{q}_{i} \dot{q}_{j},
$$

the double appearance of $i$ and $j$ denoting summation from 1 to $n$. The $a_{i j}$ are functions of $q_{1}, \cdots, q_{n}$ and we may suppose that $a_{i j}=a_{j i}$. Let the kinetic potential be

$$
L=T+F\left(q_{1}, \cdots, q_{n}\right),
$$

and let

$$
D_{q_{i}}=\frac{d}{d t}\left(\frac{\partial}{\partial \dot{q}_{i}}\right)-\frac{\partial}{\partial q_{i}} .
$$

With this notation Lagrange's equations are

$$
D_{q_{i}} L=0 \text {. }
$$

Suppose that the $q_{i}$ are related to $n+1$ new variables, $r_{1}, \cdots, r_{n+1}$, by the equations

$$
q_{i}=q_{i}\left(r_{1}, \cdots, r_{n+1}\right), \quad(i=1,2, \cdots, n),
$$

which are such that

$$
\frac{\partial\left(q_{1}, \cdots, q_{n}\right)}{\lambda\left(r_{1}, \cdots, r_{n}\right)} \not \equiv 0
$$


We have

$$
\dot{q}_{i}=\frac{\partial q_{i}}{\partial r_{s}} \dot{r}_{s}, \quad(s=1,2, \cdots, n+1)
$$

and

$$
\frac{\partial \dot{q}_{i}}{\partial \dot{r}_{s}}=\frac{\partial q_{i}}{\partial r_{s}}
$$

When $L$ is expressed in terms of $r_{s}, \dot{r}_{s}$, we shall write it $L^{\prime}$. Now

Since

$$
\begin{aligned}
\frac{\partial L^{\prime}}{\partial \dot{r}_{s}} & =\frac{\partial L}{\partial \dot{q}_{i}} \frac{\partial \dot{q}_{i}}{\partial \dot{r}_{s}}=\frac{\partial L}{\partial \dot{q}_{i}} \frac{\partial q_{i}}{\partial r_{s}} \\
\frac{d}{d t}\left(\frac{\partial L^{\prime}}{\partial \dot{r}_{s}}\right) & =\frac{\partial q_{i}}{\partial r_{s}} \frac{d}{d t}\left(\frac{\partial L}{\partial \dot{q}_{i}}\right)+\frac{\partial L}{\partial \dot{q}_{i}} \frac{d}{d t}\left(\frac{\partial q_{i}}{\partial r_{s}}\right), \\
\frac{\partial L^{\prime}}{\partial r_{s}} & =\frac{\partial L}{\partial q_{i}} \frac{\partial q_{i}}{\partial r_{s}}+\frac{\partial L}{\partial \dot{q}_{i}} \frac{\partial \dot{q}_{i}}{\partial r_{s}} .
\end{aligned}
$$

$$
\frac{\partial \dot{q}_{i}}{\partial r_{s}}=\frac{d}{d t}\left(\frac{\partial q_{i}}{\partial r_{s}}\right)
$$

we have

$$
D_{r_{s}} L^{\prime}=\frac{\partial q_{i}}{\partial r_{s}} D_{q_{i}} L
$$

Since $D_{q_{i}} L=0$ along the trajectories, we have

$$
D_{r_{s}} L^{\prime}=0
$$

along these trajectories. But as we should expect, these equations are not sufficient to determine $r_{1}, \cdots, r_{n+1}$. In fact the equations are not independent, for it can be verified that

$$
\sum_{s=1}^{n+1}(-1)^{s} \frac{\partial\left(q_{1}, \cdots, q_{n}\right)}{\partial\left(r_{1}, \cdots, r_{s-1}, r_{s+1}, \cdots, r_{n+1}\right)} D_{r_{s}} L^{\prime}=0 .
$$

Let us restrict the variables $r_{1}, \cdots, r_{n+1}$ by an equation of the form

$$
\alpha_{s} \dot{r}_{s}=0, \quad\left(s=1,2, \cdots, n+1, \alpha_{n+1} \neq \equiv\right),
$$

where the $\alpha$ 's are functions of $r_{1}, \cdots, r_{n+1}$. This enables us to eliminate $\dot{r}_{n+1}$ from $L^{\prime}$. After this elimination the kinetic po- 
tential will be denoted by $L^{\prime \prime}$. We note that $L=L^{\prime}=L^{\prime \prime}$, but that the partial derivatives of the last two are not in general equal.

If the subscript zero denotes that after differentiation $\dot{r}_{n+1}$ is eliminated by means of (14), then if $s \neq n+1$,

$$
\begin{aligned}
& \frac{\partial L^{\prime \prime}}{\partial \dot{r}_{s}}=\left(\frac{\partial L^{\prime}}{\partial \dot{r}_{s}}\right)_{0}+\left(\frac{\partial L^{\prime}}{\partial \dot{r}_{n+1}}\right)_{0} \frac{\partial \dot{r}_{n+1}}{\partial \dot{r}_{s}}, \\
& \frac{\partial L^{\prime \prime}}{\partial r_{s}}=\left(\frac{\partial L^{\prime}}{\partial r_{s}}\right)_{0}+\left(\frac{\partial L^{\prime}}{\partial \dot{r}_{n+1}}\right)_{0} \frac{\partial \dot{r}_{n+1}}{\partial r_{s}} .
\end{aligned}
$$

Hence,

$$
D_{r_{s}} L^{\prime \prime}=\left(D_{r_{s}} L^{\prime}\right)_{0}+\frac{d}{d t}\left[\left(\frac{\partial L^{\prime}}{\partial \dot{r}_{n+1}}\right)_{0} \frac{\partial \dot{r}_{n+1}}{\partial \dot{r}_{s}}\right]-\left(\frac{\partial L^{\prime}}{\partial \dot{r}_{n+1}}\right)_{0} \frac{\partial \dot{r}_{n+1}}{\partial r_{s}} .
$$

Let us suppose that (14) is so chosen that

$$
\left(\frac{\partial L^{\prime}}{\partial \dot{r}_{n+1}}\right)_{0} \equiv 0 \text {. }
$$

It will be seen by Theorem $2 \mathrm{~A}$ that this assumption is equivalent to saying that (14) is identical with

$$
\frac{\partial L^{\prime}}{\partial \dot{r}_{n+1}}=0 .
$$

From (15) and (16) it follows that

$$
D_{r_{s}} L^{\prime \prime}=0
$$

along the trajectories. It is easily shown that this equation also holds for $s=n+1$, in which case it becomes $\partial L^{\prime \prime} / \partial r_{n+1}=0$.

If we replace $L^{\prime}$ by $L^{\prime \prime}$ in (13), this equation holds by virtue of (15) and (16). It is no longer an identity, however, since it holds only by virtue of (16), that is, of (14). In other words (14) is deducible from equations (17) and may be used in place of any one of these. We can now state the following theorem.

Theorem 1. If $L\left(q_{1}, \cdots, q_{n}, \dot{q}_{1}, \cdots, \dot{q}_{n}\right)$ is the kinetic potential in a conservative holonomic dynamical system and we put

$$
q_{i}=q_{i}\left(r_{1}, \cdots, r_{n+1}\right)
$$


and suppose that

$$
\frac{\partial L^{\prime}}{\partial \dot{r}_{n+1}}=0
$$

then $r_{1}, \cdots, r_{n+1}$ are determined by the equations

$$
D_{r_{s}} L^{\prime \prime}=0
$$

and $\left(14^{\prime}\right)$ is a consequence of these equations.

3. Alternative Conditions to Replace (16).

TheOREM 2. Condition (16) implies and is implied by either of the following:

(A). The ratio

$$
\alpha_{s}: a_{i j} \frac{\partial q_{i}}{\partial r_{s}} \frac{\partial \dot{q}_{j}}{\partial r_{n+1}}
$$

is the same for every $s=1,2, \cdots, n+1$.

(B). If $\dot{r}_{n+1}$ is eliminated from $\dot{q}_{i}$ by (14),

$$
a_{i j} \frac{\partial q_{j}}{\partial r_{n+1}} \dot{q}_{i} \equiv 0 \text {. }
$$

The proof of these conditions is immediate. Since $L$ contains $\dot{q}_{i}$ and hence $\dot{r}_{s}$ only through $T,(16)$ becomes

$$
\left(\frac{\partial T^{\prime}}{\partial \dot{r}_{n+1}}\right)_{0} \equiv 0
$$

By (6) and (9) we have

so that

$$
2 T^{\prime}=a_{i j} \frac{\partial q_{i}}{\partial r_{s}} \frac{\partial q_{j}}{\partial r_{\sigma}} \dot{r}_{s} \dot{r}_{\sigma}, \quad(s, \sigma=1,2, \cdots, n+1),
$$

and

$$
\begin{array}{r}
\left(\frac{\partial T^{\prime}}{\partial \dot{r}_{n+1}}\right)_{0}=a_{i j} \frac{\partial q_{j}}{\partial r_{n+1}}\left[\frac{\partial q_{i}}{\partial r_{k}}-\frac{\alpha_{k}}{\alpha_{n+1}}-\frac{\partial q_{i}}{\partial r_{n+1}}\right] \dot{r}_{k} \equiv 0, \\
(k=1, \cdots, n) .
\end{array}
$$


From this it follows that

$$
a_{i j} \frac{\partial q_{j}}{\partial r_{n+1}}\left[\frac{\partial q_{i}}{\partial r_{k}}-\frac{\alpha_{k}}{\alpha_{n+1}} \frac{\partial q_{i}}{\partial r_{n+1}}\right] \equiv 0, \quad(k=1, \cdots, n),
$$

and hence

$$
\alpha_{k}: a_{i j} \frac{\partial q_{i}}{\partial r_{k}} \frac{\partial q_{j}}{\partial r_{n+1}}=\alpha_{n+1}: a_{i j} \frac{\partial q_{i}}{\partial r_{n+1}} \frac{\partial q_{j}}{\partial r_{n+1}},
$$

showing that the ratio on the left is the same for all values of $k$. Conversely if (20) is satisfied then (19) follows and hence (18), or (16). This proves the equivalence of (A) and (16).

When $\dot{r}_{n+1}$ is eliminated from $\dot{q}_{i}$ by (14) we get

$$
\dot{q}_{i}=\left[\frac{\partial q_{i}}{\partial r_{k}}-\frac{\alpha_{k}}{\alpha_{n+1}} \frac{\partial q_{i}}{\partial r_{n+1}}\right] \dot{r}_{k}, \quad(k=1, \cdots, n),
$$

and hence by (18)

$$
a_{i j} \frac{\partial q_{j}}{\partial r_{n+1}} \dot{q}_{i} \equiv 0
$$

This proves the equivalence of (B) and (16).

THEOREM 3. If (16) is satisfied, then when $\dot{r}_{n+1}$ is eliminated from $\dot{q}_{1}, \cdots, \dot{q}_{n}$,

$$
\frac{\partial\left(\dot{q}_{1}, \cdots, \dot{q}_{n}\right)}{\partial\left(\dot{r}_{1}, \cdots, \dot{r}_{n}\right)} \equiv 0 .
$$

Equations (19) may be regarded as $n$ equations for the determination of $\partial q_{j} / \partial r_{n+1}$. Since these derivatives do not all vanish and the equations defining them are homogeneous, it follows that the determinant

$$
\left|b_{j k}\right| \equiv\left|a_{i j}\left(\frac{\partial q_{i}}{\partial r_{k}}-\frac{\alpha_{k}}{\alpha_{n+1}} \frac{\partial q_{i}}{\partial r_{n+1}}\right)\right|
$$

is zero. But by the rule for multiplying determinants

$$
\begin{array}{rlr}
\left|b_{j k}\right| & =\left|a_{i j}\right|\left|\frac{\partial q_{i}}{\partial r_{k}}-\frac{\alpha_{k}}{\alpha_{n+1}} \frac{\partial q_{i}}{\partial r_{n+1}}\right|, & \\
& =\left|a_{i j}\right|\left|\frac{\partial \dot{q}_{i}}{\partial \dot{r}_{k}}\right|, &
\end{array}
$$


by (21). Since $\left|a_{i j}\right|$ is the determinant of a positive quadratic form, we must have

$$
\left|\frac{\partial \dot{q}_{i}}{\partial \dot{r}_{k}}\right|=\frac{\partial\left(\dot{q}_{1}, \cdots, \dot{q}_{n}\right)}{\partial\left(\dot{r}_{1}, \cdots, \dot{r}_{n}\right)} \equiv 0 .
$$

4. Geometric Aspects of the Theory. In this section we confine ourselves to the case $n=2$. The discussion will also apply to the case in which all but two of the $q_{i}$ are not changed by the transformation. If

$$
2 T=a_{11} \dot{q}_{1}^{2}+2 a_{12} \dot{q}_{1} \dot{q}_{2}+a_{22} \dot{q}_{2}^{2},
$$

then $T$ may be regarded as the kinetic energy of a particle of unit mass constrained to move in a surface whose metric is

$$
d s^{2}=a_{11} d q_{1}^{2}+2 a_{12} d q_{1} d q_{2}+a_{22} d q_{2}^{2} .
$$

At any point $P$ of the path $\pi$ of the particle in this surface construct a geodesic $G$ tangent to $\pi$. Let $G$ cut any fixed directed curve $\Gamma$ at $N$, and let $O$ be a fixed point of $\Gamma$. Let $r_{1}=O N$, measured along $\Gamma, r_{2}=N P$, measured along $G, r_{3}=$ angle between $\Gamma$ and $G$. The equations of $\pi, q_{i}=q_{i}(t)$, are found by solving the given dynamical problem, while $\dot{q}_{i}$ are direction numbers of the tangent to $\pi$. The relations between $q_{i}$ and $r_{3}$ are

$$
q_{i}=q_{i}\left(r_{1}, r_{2}, r_{3}\right), \quad(i=1,2) .
$$

If $r_{1}$ and $r_{2}$ are held fast, these are the equations of a geodesic circle, $C$, with center at $N$, and $\partial q_{i} / \partial r_{3}$ are direction numbers of the tangent to $C$. Now $C$ is perpendicular to $G$ and hence also to $\pi$. The latter fact is expressed by the equation

$$
a_{i j} \dot{q}_{i} \frac{\partial q_{j}}{\partial r_{3}} \equiv 0 .
$$

By Theorem 2B this is a necessary and sufficient condition that

$$
\left(\frac{\partial T^{\prime}}{\partial \dot{r}_{3}}\right)_{0}=0 \text {. }
$$

Conversely if (26) holds, $G$ will be tangent to $\pi$. These results may be stated as follows: 
THEOREM 4. If the kinetic energy of a dynamical system is given by

$$
2 T=a_{i j} \dot{q}_{i} \dot{q}_{j}, \quad(i, j=1,2),
$$

and if on a surface whose metric is

$$
d s^{2}=a_{i j} d q_{i} d q_{j}, \quad(i, j=1,2),
$$

$r_{1}, r_{2}, r_{3}$ are three coordinates chosen in the manner specified above, then

$$
\left(\frac{\partial T^{\prime}}{\partial \dot{r}_{3}}\right)_{0} \equiv 0
$$

Also, $r_{1}, r_{2}, r_{3}$ are obtained from the equations

$$
\frac{d}{d t}\left(\frac{\partial L^{\prime \prime}}{\partial \dot{r}_{s}}\right)-\frac{\partial L^{\prime \prime}}{\partial r_{s}}=0, \quad(s=1,2,3),
$$

where the double prime denotes that the kinetic potential is expressed in terms of $r_{1}, r_{2}, r_{3}, \dot{r}_{1}, \dot{r}_{2}$.

5. Examples. For a unit particle constrained to move in the $x y$-plane, we have $2 T=\dot{x}^{2}+\dot{y}^{2}$. Let $P$ be the point $(x, y), N$ a point on the $x$-axis, and $O$ the origin. Put $z=O N, \rho=N P$, $\phi=X N P$. Then

$$
x=z+\rho \cos \phi, \quad y=\rho \sin \phi .
$$

If $N P$ is to be tangent to the path of the particle, we must have

$$
\dot{x} \frac{\partial x}{\partial \phi}+\dot{y} \frac{\partial y}{\partial \phi}=0 \text {. }
$$

This is the condition expressed by (25). In terms of the new variables this condition becomes

$$
\dot{z} \sin \phi-\rho \dot{\phi}=0 \text {. }
$$

We easily find

and

$$
2 T^{\prime}=\dot{z}^{2}+2 \cos \phi \dot{z} \dot{\rho}+\dot{\rho}^{2}-2 \rho \sin \phi \dot{z} \dot{\phi}+\rho^{2} \dot{\phi}^{2},
$$

$$
\frac{\partial T^{\prime}}{\partial \dot{\phi}}=-\rho \sin \phi \dot{z}+\rho^{2} \dot{\phi}=0,
$$

by (27). Using (27) we get

$$
2 T^{\prime \prime}=(\dot{z} \cos \phi+\dot{\rho})^{2} .
$$


It may be remarked that since (25) is linear and homogeneous in $\dot{q}_{i}, T^{\prime \prime}$ will always be a perfect square when $n=2$. If $F$ is the force function expressed in terms of $z, \rho, \phi$, the equations of motion are, by Theorem 4 ,

$$
\begin{aligned}
\frac{d}{d t}[\cos \phi(\dot{z} \cos \phi+\dot{\rho})] & =\frac{\partial F}{\partial z}, \\
\frac{d}{d t}(\dot{z} \cos \phi+\dot{\rho}) & =\frac{\partial F}{\partial \rho}, \\
\dot{z} \sin \phi(\dot{z} \cos \phi+\dot{\rho}) & =\frac{\partial F}{\partial \phi} .
\end{aligned}
$$

If we multiply these equations by $\rho,-\rho \cos \phi, \sin \phi$ respectively and add we obtain (27).

The total order of these equations appears to be five, but it is in reality only four since a first integral can be obtained from a linear combination of them without any integration. If the reader wishes to carry through the integration for the case $F=-g y=-g \rho \sin \phi$, he will see that only four arbitrary constants enter. The force function suggested is that due to gravity.

A second example is that given in the opening section. A careful comparison of this example with the theory is suggested.

6. Case of more than One Extra Coordinate. The preceding theory can be extended to the case in which more than one additional coordinate is introduced. If the equations of transformation are

$$
q_{i}=q_{i}\left(r_{1}, \cdots, r_{n+p}\right), \quad(i=1, \cdots, n),
$$

there are $p$ extra variables. If the new variables are restricted by $p$ equations like (14), and if as a consequence of these relations we have

$$
\left(\frac{\partial T^{\prime}}{\partial r_{s}}\right)_{0} \equiv 0, \quad(s=n+1, \cdots, n+p),
$$

then it can be shown that

$$
D_{r_{s}} L^{\prime \prime}=0, \quad(s=1,2, \cdots, n+p) .
$$


No satisfactory geometrical interpretation has been found except for the case $p=n-1$. This includes also the case $p<n-1$, provided that $n-p-1$ of the original coordinates are not affected by the transformation. For example, in the case discussed in the first section $n=3$ and $p=1$ and there was one coordinate not affected by the transformation. The geometrical interpretation for $p=n-1$ is similar to that for $n=2, p=1$. If

$$
2 T=a_{i j} \dot{q}_{i} \dot{q}_{j}, \quad(i, j=1,2, \cdots, n),
$$

then $T$ may be regarded as the kinetic energy of a unit particle moving in a space of $n$ dimensions whose metric is

$$
d s^{2}=a_{i j} d q_{i} d q_{j}, \quad(i, j=1,2, \cdots, n) .
$$

Let $\left(r_{1}, \cdots, r_{n-1}, 0\right)$ represent the $q$-coordinates of a point $N$ in this space; $r_{n}=$ the distance along the geodesic joining $N$ and the point $P:\left(q_{1}, \cdots, q_{n}\right) ; r_{n+1}, \cdots, r_{2 n-1}$ be the first $n-1$ direction cosines of this geodesic at $N$. Suppose the point $N$ is so chosen that this geodesic will be tangent to the path at $P$. If then $r_{1}, \cdots, r_{n}$ are held fast in the equations of transformation,

$$
q_{i}=q_{i}\left(r_{1}, \cdots, r_{2 n-1}\right), \quad(i=1,2, \cdots, n),
$$

these equations become those of a geodesic sphere. This sphere will be perpendicular to the geodesic $N P$ at $P$, and hence also to the path. This fact is expressed by the $n-1$ equations

$$
a_{i j} \dot{q}_{i} \frac{\partial q_{j}}{\partial r_{s}}=0, \quad(s=n+1, \cdots, 2 n-1) .
$$

But these are necessary and sufficient that

$$
\left(\frac{\partial T}{\partial \dot{r}_{s}}\right)_{0} \equiv 0, \quad(s=n+1, \cdots, 2 n-1)
$$

so that equations (29) will hold.

LEHIGH UNIVERSITY 